\title{
Study of the reinforcement of structure members by polypropylene fibres waste
}

\section{Etude de renforcement des éléments de structure par un déchet de fibres de polypropylène}

\author{
Khadra Bendjillali ${ }^{1}$ and Mohamed Chemrouk ${ }^{2}$ \\ ${ }^{1}$ University Amar Telidji, Faculty of Civil Engineering and Architecture, Civil Engineering Department, Laghouat, Algeria \\ ${ }^{2}$ University Houari Boumediene, Faculty of Civil Engineering, Algiers, Algeria
}

\begin{abstract}
The valorisation of industrial waste in the field of construction became a very interesting axis of research from scientific, economic and environmental point of view. We have conducted this work to study the effect of the addition of polypropylene fibres waste on the mechanical behaviour of reinforced concrete beams subjected to a simple flexural loading, with and without transversal reinforcement. The used fibres are coming from the waste of the fabrication of domestic brushes and sweeps; they have an average diameter of $0.47 \mathrm{~mm}$ and a length between 40 and $60 \mathrm{~mm}$. Two weight dosages of fibres are used, 0.25 and $0.5 \%$. The experimental results showed that the incorporation of polypropylene fibres waste into the concrete affects negatively its workability, but its flexural and compressive strength are improved. The fibers have presented a significant contribution on the shear behavior and the cracking of beams, particularly in absence of transversal bars. The waste used in this work as fibrous reinforcement has not only increased the ductility of reinforced concrete beams, but it have also provided a perfect cracking distribution on the concrete surface and it has participated in a considerable way in the reduction of cracks number and dimensions, which allows to ensure the material durability and then the structure longevity. The reinforcement of concrete beams with $0.5 \%$ of polypropylene fibers waste with a minimal steel bars can ensure an excellent mechanical behavior in shear, as in flexion.
\end{abstract}

Résumé. La valorisation des déchets industriels dans le domaine de la construction est devenue actuellement un axe de recherche très intéressant, non seulement du point de vue scientifique, mais encore du point de vue économique et environnemental. C'est alors dans cette optique que nous avons mené ce travail qui consiste à étudier l'effet de l'addition d'un déchet en fibres de polypropylène sur le comportement mécanique des poutres en béton armé soumises à la flexion simple en présence ou non du ferraillage transversal. Le renforcement employé est un déchet fibreux provenant de la fabrication des balais et des brosses domestiques, de diamètre moyen de l'ordre de $0.47 \mathrm{~mm}$ et de longueur variant entre 40 et $60 \mathrm{~mm}$. Deux dosages massiques de fibres sont employés 0.25 et $0.5 \%$. Les résultats expérimentaux ont montré que bien que l'incorporation du déchet en fibres de polypropylène dans le béton a affecté négativement l'ouvrabilité du matériau, mais leur effet sur sa résistance à la flexion et à la compression est très considérable. Les fibres employées ont présenté également un apport très significatif sur le comportement au cisaillement des éléments poutres, particulièrement en absence d'armatures transversales et aussi sur leur état de fissuration. Le déchet utilisé comme renforcement fibreux non seulement a augmenté la ductilité des poutres en béton armé, mais il a aussi offert une parfaite distribution des fissures sur la surface du béton et il a participé d'une façon très appréciable à la réduction du nombre de fissures et de leur dimensions, ce qui permettra d'assurer une durabilité du matériau et par la suite une longévité de la structure. Le renforcement des poutres en béton avec un pourcentage massique de $0.5 \%$ de déchet de fibres de polypropylène avec un minimum d'armatures d'acier peut leur assurer un excellent comportement mécanique au cisaillement, comme en flexion. 


\section{Introduction}

Le mode de rupture et la fissuration des poutres en béton armé dépendent de leurs dimensions, du mode de chargement, des propriétés mécaniques du béton et de l'acier et de la quantité et le type du ferraillage employé. Si la rupture par flexion de ces poutres est bien maitrisée et bien prise en compte par les différents textes normatifs, la rupture par effort tranchant plus complexe demeure mal appréhendée. Des armatures transversales sont disposées pour reprendre une partie des contraintes de cisaillement et coudre les fissures d'effort tranchant. La présence de fibres dans les poutres peut aider le béton notamment dans les zones les plus sollicitées et ainsi soulager le ferraillage conventionnel. La question qui se pose actuellement est la suivante: peut-on substituer ce ferraillage en acier par des fibres? Cette question est d'un intérêt économique énorme car avec moins d'armatures transversales, non seulement le prix de la construction est réduit, mais aussi le temps d'exécution [1]. Certains auteurs [2,3] ont trouvé que le comportement mécanique des poutres renforcées par des fibres et sans ferraillage conventionnel est meilleur que le comportement des poutres sans fibres et avec des armatures transversales. Selon Halvax et Lublóy [1], les performances des poutres renforcées uniquement de fibres d'acier sont pratiquement identiques à celles des poutres avec uniquement un ferraillage transversal, avec une légère augmentation de la ductilité et de la charge ultime de cisaillement dans le cas des poutres renforcées de fibres d'acier. Selon les mêmes auteurs, la rupture par cisaillement des poutres peut être évitée en incorporant un volume de $1 \%$ de fibres d'acier. Dans une autre étude [4], il était constaté que les fibres d'acier sont capables de contrôler parfaitement la fissuration, sans influer la ductilité et la résistance du matériau. La chose la plus sûre selon toutes les études de la littérature et relatives à ce sujet, est que les fibres sont capables de retarder la fissuration engendrée par l'effort tranchant et de contrôler son ouverture. Cependant, leur effet sur le mode de rupture et sur la résistance à l'arrachement est conditionné par plusieurs paramètres, tels que leurs caractéristiques géométriques et leurs dosages, leurs orientations et leur adhérence avec la matrice cimentaire. Selon certains chercheurs $[1,4,5]$, les fibres ne peuvent pas remplacer complètement le ferraillage transversal, mais elles sont capables de remplacer une partie de sa section, notamment dans les zones nodales, où il y'a trop d'aciers qui constituent un obstacle pour le passage du béton, ce qui empêche d'avoir un bon remplissage des coffrages. L'emploi de fibres de polypropylène dans le béton a connu ces dernières années une grande évolution grâce à leurs performances, telles que la légèreté, la non absorptivité d'eau, la non réactivité avec le ciment et la facilité de dispersion dans la matrice. Les fibres de polypropylène améliorent la ductilité du béton, réduisent significativement la largeur des fissures [6] et peuvent retarder la dégradation des structures en réduisant la perméabilité, le retrait et l'expansion du béton [7].

Dans ce travail expérimental, nous allons étudier l'effet du renforcement des poutres en béton armé par un déchet de fibres de polypropylène issu de l'industrie des brosses et des balais ménagères sur leur comportement mécanique et leur état de fissuration. Nous allons tester ce comportement en présence ou non d'armatures transversales, pour voir si nous pouvons remplacer le ferraillage conventionnel par des fibres. Par ailleurs, l'emploi d'un déchet de fibres comme renforcement dans les structures en béton est une tentative de recyclage de déchet afin de réduire le coût de la construction et protéger notre environnement.

\section{Programme experimental}

\subsection{Matériaux}

Le béton utilisé dans la présente étude est à base de:

Ciment portland composé CEM II/A-L 42.5R (C) importé du Portugal, ayant une finesse de Blaine de 3520 $\mathrm{cm}^{2} / \mathrm{g}$ et une densité absolue de 3.13 .

Eau de gâchage (E) provenant du réseau de distribution d'eau potable.

Granulats calcaires de concassage, un sable (S) 0/3 mm et deux graviers (G1) $3 / 8 \mathrm{~mm}$ et (G2) $8 / 15 \mathrm{~mm}$ provenant de la station de concassage Ouazane de Laghouat, de densité absolue au voisinage de 2.50 et de coefficient d'absorption égal respectivement $4.71,1.50$ et $1.80 \%$. Les courbes granulométriques des granulats utilisés sont présentées sur la figure 1 . Le sable possède un équivalent de sable de l'ordre de $66 \%$.

Déchet de fibres synthétiques en polypropylène $(\mathrm{F})$ de diamètre moyen de $0.47 \mathrm{~mm}$, de longueur variant entre 40 et $60 \mathrm{~mm}$ et de résistance à la traction de l'ordre de $218 \mathrm{MPa}$ (figure 2).

Superplastifiant (SP) du nom commercial SIKAVISCOCRETE TEMPO 12 fabriqué par la société SIKA EL DJAZAIR.

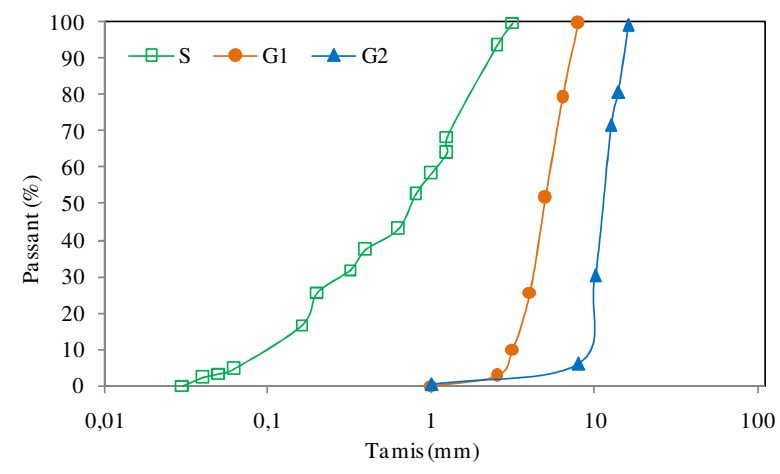

Fig. 1. Distribution granulaire des granulats.

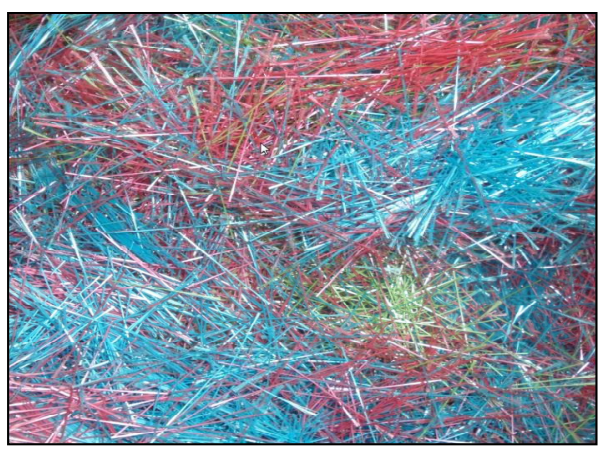


Fig. 2. Déchet de fibres de polypropylène.

\subsection{Formulation et préparation des échantillons}

Les compositions du béton témoin sans fibres B0 et les deux bétons fibrés B0.25 et B0.5 renfermant respectivement un dosage massique de 0.25 et $0.5 \%$ de fibres sont données dans le tableau 1. Les rapports (E/C) et $(\mathrm{SP} / \mathrm{C})$ pour ces trois bétons sont constants et valent respectivement 0.57 et $1.4 \%$. La formulation du béton témoin a été déterminée par la méthode pratique de Dreux-Gorisse [8]. Les composants secs sont mis en premier dans une bétonnière et après un bon malaxage, nous ajoutons la moitié de la quantité d'eau mélangée avec le superplastifiant, puis la deuxième moitié qui reste. Les fibres sont introduites manuellement en dernier afin d'assurer une bonne dispersion et d'éviter la formation des oursins. Nous avons confectionné des échantillons cubiques 10x10x10 $\mathrm{cm}$ pour l'essai de compression simple, la valeur retenue est la moyenne de six résultats et d'autres échantillons prismatiques $7 \times 7 \times 28 \mathrm{~cm}$ pour l'essai de flexion à trois points, dont la résistance est calculée par la moyenne de trois valeurs. Des poutres 10x10x50 cm avec le ferraillage schématisé sur la figure 3 sont également confectionnées. Les échantillons de béton sont démoulés 24 heures après leur confection puis ils sont conservés sous la toile de jute mouillée à une température variant entre 20 et $30{ }^{\circ} \mathrm{C}$ jusqu'au jour de l'essai à 28 jours d'âge du béton.

Tableau 1. Composition des bétons $\left(\mathrm{kg} / \mathrm{m}^{3}\right)$.

\begin{tabular}{|c|c|c|c|c|c|c|c|}
\hline Béton & C & E & S & G1 & G2 & F & SP \\
\hline B0 & 350 & 199.5 & 670 & 186 & 899 & 0 & 4.9 \\
\hline B0.25 & 350 & 199.5 & 670 & 186 & 899 & 5.8 & 4.9 \\
\hline B0.5 & 350 & 199.5 & 670 & 186 & 899 & 11.6 & 4.9 \\
\hline
\end{tabular}

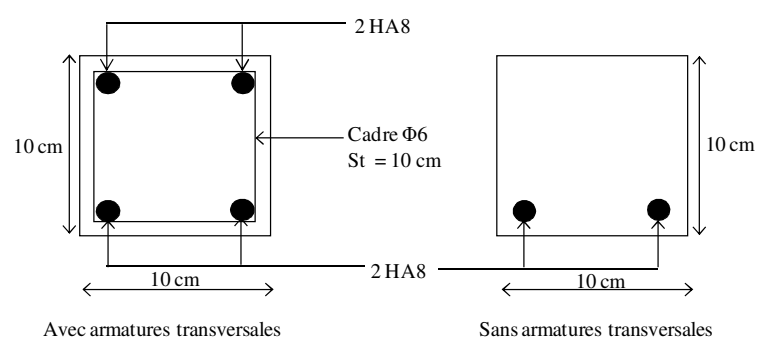

Fig. 3. Ferraillage des poutres.

\section{Résultats expérimentaux}

\subsection{Maniabilité}

La maniabilité du béton diminue avec l'augmentation du dosage en fibre de polypropylène. En effet, le béton témoin B0 présente un affaissement mesuré au cône
d'Abrams de $9.3 \mathrm{~cm}$, alors que les bétons de fibres B0.25 et B0.5 présentent respectivement un affaissement de 6 et $1 \mathrm{~cm}$ (figure 4). Avec la présence de fibres de polypropylène, le béton devient plus ferme et sa mise en œuvre plus délicate. Ainsi afin de maintenir une bonne plasticité du béton et une bonne distribution des fibres, l'augmentation du dosage de fibres nécessite une augmentation du dosage de superplastifiant. Pour cette raison, nous avons limité le dosage massique de fibres à $0.5 \%$ afin d'éviter la formation d'oursins (figure 5) qui constituent un problème très délicat dans la fabrication des bétons de fibres. Les études de la littérature [9-13] montrent que l'addition des fibres dans les matrices cimentaires engendre une réduction de leur plasticité.

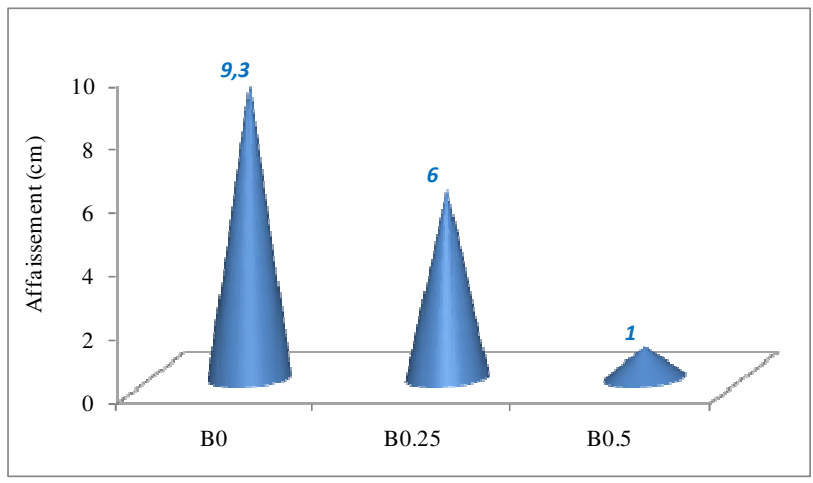

Fig. 4. Affaissement au cône d'Abrams des bétons étudiés.

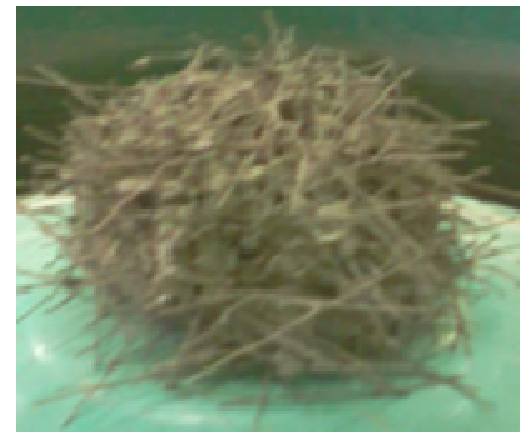

Fig. 5. Oursins formés.

\subsection{Comportement à la flexion}

Le comportement des bétons en flexion s'améliore avec l'addition de fibres de polypropylène (figure 6). L'amélioration augmente avec le dosage de fibres. Par rapport au béton témoin sans fibres B0, les bétons renforcés par 0.25 et $0.5 \%$ en masse de fibres conduisent à des augmentations de la résistance à la traction par flexion respectivement de l'ordre de 9 et $30 \%$. Les fibres n'interviennent qu'après la rupture du béton, elles transfèrent les contraintes à travers la fissure, en offrant ainsi au matériau une certaine rigidité et un contrôle parfait de l'ouverture des fissures. La majorité des recherches faites sur les matériaux cimentaires renforcés de fibres [14-17] confirment l'efficacité des fibres sur le comportement en flexion des matériaux. Nous avons constaté que l'effet des fibres sur le comportement en flexion est conditionné par leur distribution efficace dans le béton. Certains chercheurs [17] confirment qu'une 
mauvaise distribution des fibres conduit à une réduction de cette résistance.

\subsection{Comportement à la compression}

La résistance à la compression des bétons à 28 jours augmente également avec l'ajout de déchet de fibres de polypropylène (figure 7). Cette augmentation est moins importante que celle observée avec la résistance à la traction par flexion puisqu'elle varie entre 2 et $16 \%$ respectivement pour les bétons B 0.25 et B0.5 par rapport au béton témoin sans fibres $\mathrm{B} 0$. Ce résultat est concordant avec d'autres travaux [18-21], même si d'autres auteurs ont constaté une réduction de la résistance à la compression après l'ajout de fibres [22-25]. L'effet positif des fibres de polypropylène sur le comportement à la compression peut être expliqué par leur flexibilité et leur souplesse qui leur permettent de se loger facilement entre les granulats sans les perturber et par conséquent d'augmenter la compacité du matériau, et donc améliorer son comportement en compression. Certains auteurs [26] voient que l'efficacité des fibres sur la résistance à la compression dépend de la géométrie et du dosage de fibres et non de leur type.

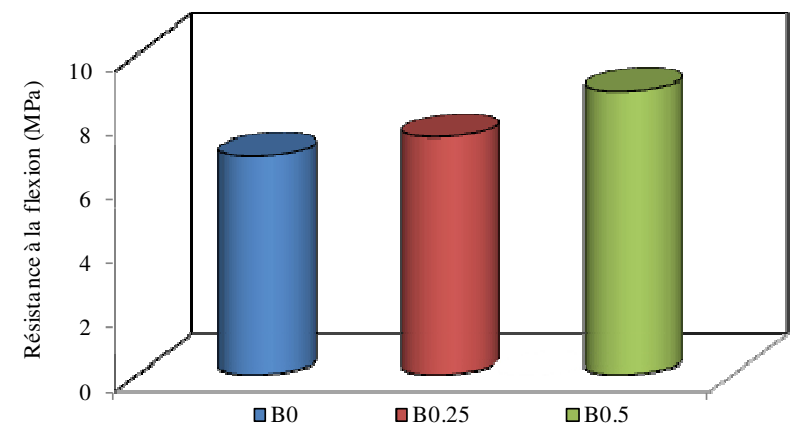

Fig. 6. Résistance à la flexion à 28 jours des bétons.

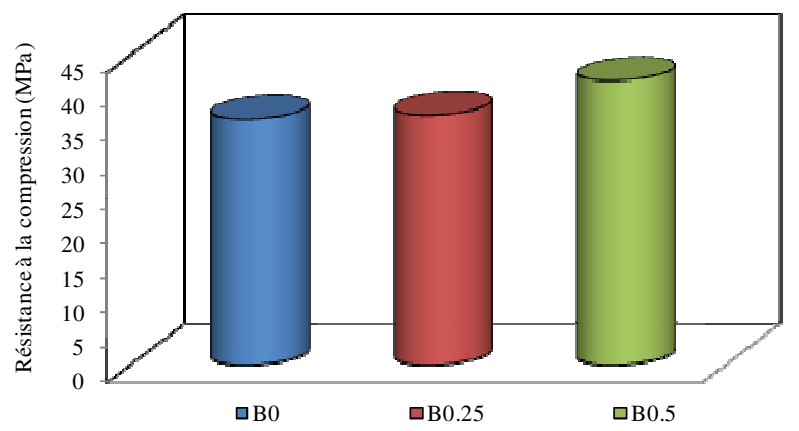

Fig. 7. Résistance à la compression à 28 jours des bétons.

\subsection{Comportement mécanique des poutres}

Dans les poutres en béton témoin sans fibres B0, la rupture se fait par cisaillement si aucune armature transversale n'est prévue (figure 8-a). Mais avec la présence de déchet de fibres de polypropylène, aucune fissure d'effort tranchant n'est observée sur les poutres, même en absence du ferraillage transversal (figure 8-b). L'apport de déchet de fibres de polypropylène est beaucoup plus apprécié en l'absence d'armatures transversales, du faite que dans ce cas, le renforcement au cisaillement est assuré par les fibres seules qui ont présenté une grande capacité de distribution de contraintes et donc une aptitude de déformation et d'absorption d'énergie plus élevée. La même constatation était faite par d'autres chercheurs [27]. Des fissures de flexion sont localisées au voisinage de la mi-portée des poutres et qui sont plus fines en présence des armatures transversales (figures 8-c, 8-d). La rupture des poutres est alors due au moment fléchissant.

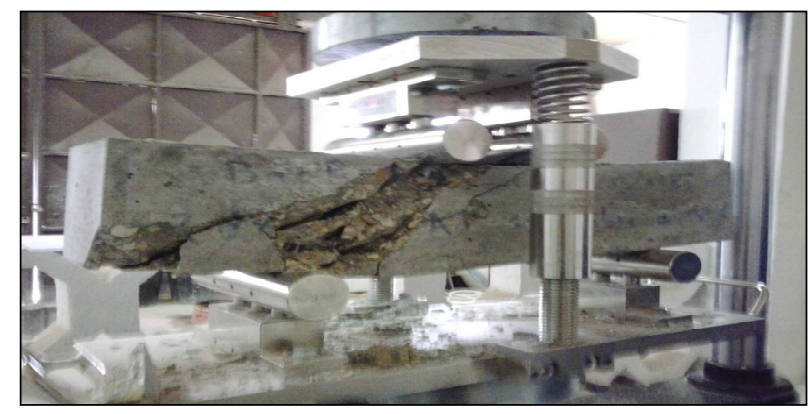

a. Poutres en B0 sans ferraillage transversal.

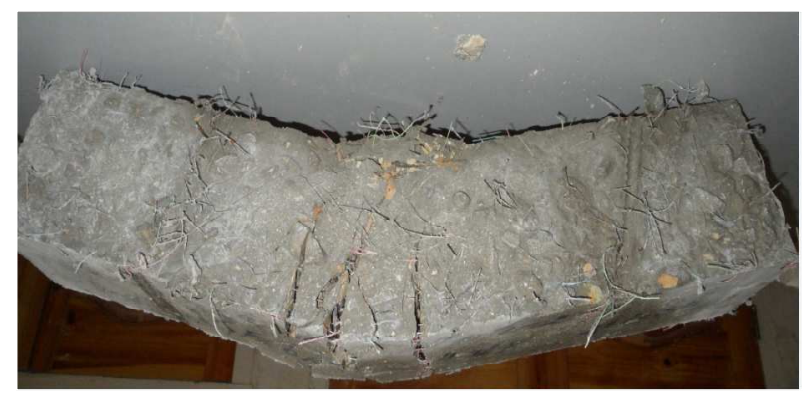

b. Poutres en B0.50 sans ferraillage transversal.

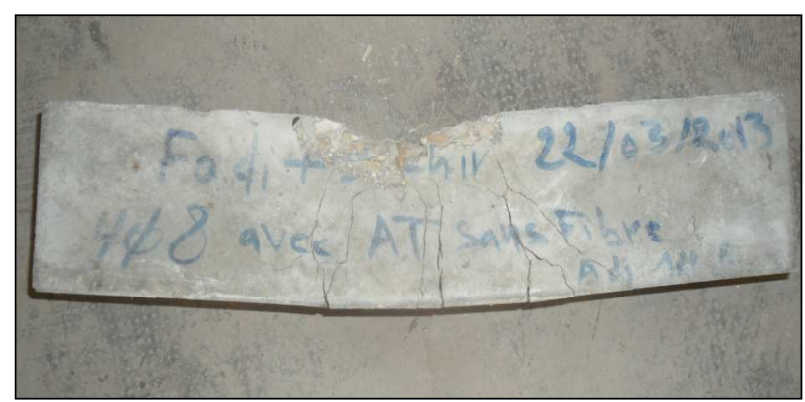

c. Poutres en B0 avec ferraillage transversal.

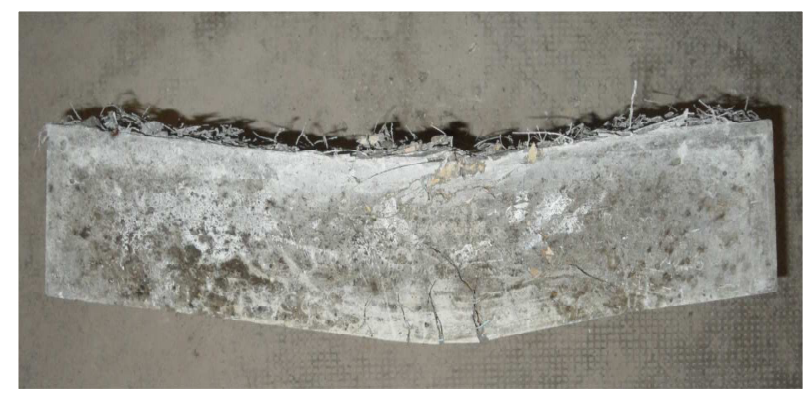

d. Poutres en B0.50 avec ferraillage transversal.

Fig. 8. Mode de rupture des poutres.

Les figures 9 et 10 schématisent la variation des valeurs du taux d'amélioration de la résistance à la 
flexion des poutres en béton de fibres respectivement à la rupture et à la première fissure. Nous pouvons clairement observer que le déchet de fibres de polypropylène a un effet positif sur le comportement mécanique des poutres même sans ferraillage transversal. Le taux d'amélioration du comportement des poutres à la rupture apporté par un dosage massique de $0.25 \%$ de fibres passe de $8 \%$ en présence d'armatures transversales à $17 \%$ sans armatures transversales; alors que dans les poutres renforcées par $0.5 \%$ de fibres, le taux d'amélioration varie entre 13 et $20 \%$ respectivement dans les poutres avec et sans ferraillage transversal (figure 9). Cependant qu'à la première fissure, l'amélioration varie entre 1 et $12 \%$ dans les poutres renforcées par $0.25 \%$ de fibres et entre 15 et $19 \%$ dans les poutres renforcées par $0.5 \%$ de fibres respectivement avec et sans ferraillage transversal (figure 10). En présence de fibres de polypropylène, les fissures qui sont apparues sur les poutres sont plus fines et mieux dispersées même dans les poutres sans ferraillage transversal. Dans certains travaux [28], il était constaté que les fibres n'affectent pas le processus de fissuration, mais plutôt la longueur et le nombre des fissures, qui deviennent plus courtes et multiples. Nous avons remarqué aussi un effet positif des fibres de polypropylène sur le comportement au cisaillement des poutres, qui est en présence de $0.5 \%$ de fibres et sans armatures transversales meilleur que celui des poutres sans fibres et avec des armatures transversales. Plusieurs travaux [29, 30] ont confirmé l'efficacité des fibres synthétiques à l'amélioration de la résistance des poutres au cisaillement. Furlan et de Hanai [31] ont confirmé selon leur essais expérimentaux que les poutres renforcées avec $2 \%$ de fibres d'acier et sans armatures transversales ont montré une fissuration semblable à la fissuration des poutres avec armatures transversales et sans fibres. Un bon comportement mécanique des poutres peut être obtenu avec la combinaison de fibres et un minimum d'armatures d'acier transversales.

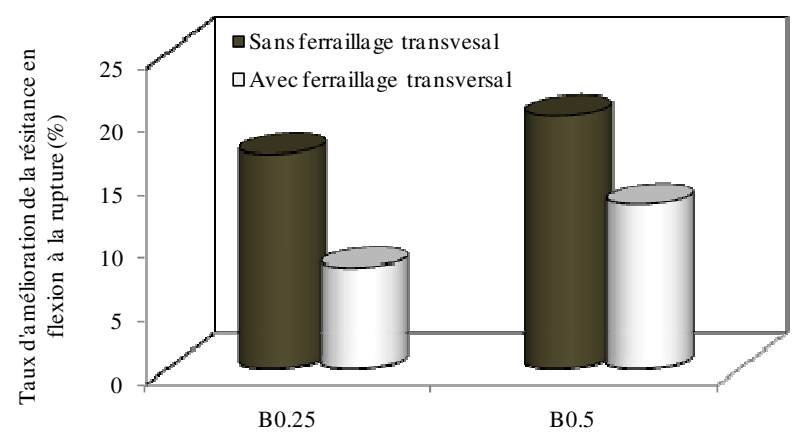

Fig. 9. Amélioration de la résistance en flexion des poutres à la rupture.

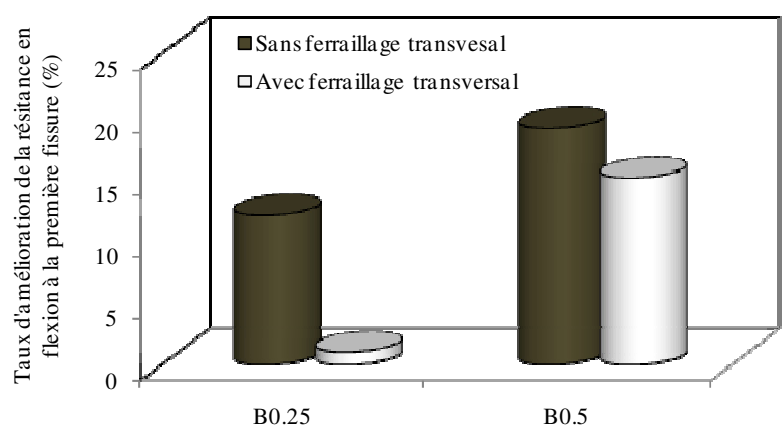

Fig. 10. Amélioration de la résistance en flexion des poutres à la première fissure.

\section{Conclusion}

À travers les résultats obtenus nous avons pu tirer les conclusions suivantes:

- L'ajout du déchet de fibres de polypropylène au béton diminue sa maniabilité.

- Les résistances à la flexion et à la compression simple du béton subissent un effet positif de l'incorporation de déchet de fibres de polypropylène. Avec un dosage massique de $0.5 \%$ en fibres, l'amélioration des résistances à la traction par flexion et à la compression est respectivement de 30 et de $16 \%$.

- L'incorporation du déchet de fibres de polypropylène dans le béton a considérablement amélioré le comportement des poutres au cisaillement même sans présence du ferraillage transversal.

- Le comportement en flexion à la rupture et à la première fissure des poutres est amélioré suite à leur renforcement par le déchet des fibres de polypropylène.

- La présence du déchet de fibres de polypropylène dans les poutres a diminué considérablement la fissuration, même sans armatures transversales.

\section{Références}

[1] K. Halvax, É. Lublóy, Steel Fiber Reinforced Concrete-Shear Strength, Acta Technica Jaurinensis, 5(2), pp 149-166, (2012).

[2] A. Meda, F. Minelli, G. A. Plizzari, P. Riva, Shear behaviour of steel fibre reinforced concrete beams, Materials \& Structures Journal, 38(3), pp 343-351, (2005).

[3] T. Nyomboi, H. Matsuda, A. Demizu, K. Makino, Experimental and analytical study on shear capacity in steel fiber and stirrup RC beam, Journal of Structural Engineering, 56A, pp 13-22, (2010).

[4] Y. Fritih, T. Vidal, A. Turatsinze, P. Gérard, Flexural and shear behavior of steel fiber reinforced SCC beams, KSCE Journal of Civil Engineering, Structural Engineering, 17(6), pp 1383-1393, (2013).

[5] D. H. Lim, B. H. Oh, Experimental and theoretical investigation on the shear of steel fibre reinforced concrete beams, Engineering Structures Journal, 21(10), pp 937-944, (1999).

[6] D. Suji, S. C. Natesan, R. Murugesan, Experimental study on behaviors of polypropylene fibrous concrete beams, Journal of Zhejiang University Science A, 8(7), pp 1101-1109, (2007).

[7] S. Kakooei, H. M. Akil, M. Jamshidi, J. Rouhi, The effects of polypropylene fibers on the properties of reinforced concrete structures, Construction \& Building Materials Journal, 27(1), pp 73-77, (2012)

[8] G. Dreux, J. Festa, Nouveaux guide du béton et de ses constituants, $8^{\text {ème }}$ ed, Eyrolles, (2002).

[9] C. D. Johnston, Fiber-reinforced cements \& concretes, Pub Gordon \& Breach Science, Amsterdam, (2001). 
[10]A. Sivakumar, M. Santhanam, A quantitative study on the plastic shrinkage cracking in high strength hybrid fibre reinforced concrete, Cement \& concrete Composites Journal, 29(7), pp 575581, (2007).

[11]H. Y. Aruntaş, S. Cemalgil, O. Şimşek, G. Durmuş, M. Erdal, Effects of super plasticizer and curing conditions on properties of concrete with and without fiber, Materials Letters Journal, 62(19), pp 3441-3443, (2008).

[12]K. Bendjillali, S. Rezzoug, M. S. Goual, M. Chemrouk, A. Zaidi, Mechanical behaviour of polypropylene fibre concrete beams, Inter Cong CMSS-2013, Rabat, Morocco, p 65, (2013).

[13]T. A. Söylev, T. Özturan, Durability, physical and mechanical properties of fiber-reinforced concretes at low-volume fraction, Construction \& Building Materials Journal, 73, pp 67-75, (2014).

[14]A. García-Santos, J. M. Rincón, M. Romero, R. Talero, Characterization of a polypropylene fibered cement composite using ESEM, FESEM and mechanical testing, Construction \& Building Materials Journal, 19(5), pp 396-403, (2005).

[15]P. Ramadoss, K. Nagamani, Tensile strength and durability characteristics of high-performance fiber reinforced concrete, The Arabian Journal for Science \& Engineering, 33(2B), pp 307-319, (2008).

[16]Z. Sun, Q. Xu, Microscopic, physical and mechanical analysis of polypropylene fiber reinforced concrete, Materials Science and Engineering A Journal, 527(1-2), pp 198-204, (2009).

[17] V. Alamshahi, A. Taeb., R. Ghaffarzadeh, M. A. Rezaee, Effect of composition and length of PP and polyseter fibres on mechanical properties of cement based composites, Construction \& Building Materials Journal, 36, pp 534-537, (2012).

[18]A. Hashem, A. Zakiah, Characterization of natural fibre concrete using destructive and non-destructive testing methods, The $6^{\text {th }}$ Inter Conf on Concrete Technology for Developing Countries, Amman, Jordan, pp 551-560, (2002).

[19]Z. J. Grdic, G. A. Toplicic Curcic, N. S. Ristic, I. M. Despotovic, Abrasion resistance of concrete micro-reinforced with polypropylene fibers, Construction \& Building Materials Journal, 27(1), pp 305-312, (2012).

[20]A. Belferrag, A. Kriker, M. E. Khenfer, Improvement of the compressive strength of mortar in the arid climates by valorization of dune sand and pneumatic waste metal fibers, Construction \& Building Materials Journal, 40, pp 847-853, (2013).

[21]H. Cifuentes, F. Garcia, O. Maeso, F. Medina, Influence of the properties of polypropylene fibres on the fracture behaviour of low, normal and high-strength FRC; Construction \& Building Materials Journal, 45, pp 130-137, (2013).

[22]A. Sadrmomtazi, A. K. Haghi, Properties of cementitious composites containing polypropylene fiber waste, Composite Interfaces Journal, 15(7-9), pp 867-879, (2008).

[23]M. S. Meddah, M. Bencheikh, Properties of concrete reinforced with different kinds of industrial waste fibre materials, Construction \& Building Materials Journal, 23(10), pp 3196-3205, (2009).

[24] A. A. Ramezanianpour, M. Esmaeili, S. A. Ghahari, M. H. Najafi, Laboratory study on the effect of polypropylene fiber on durability and physical and mechanical characteristic of concrete for application in sleepers, Construction \& Building Materials Journal, 44, pp 411-418, (2013).

[25]T. Tahenni, M. Chemrouk, T. Lecompte, Effect of steel fibers on the shear behavior of high strength concrete beams, Construction \& Building Materials Journal, 105, pp 14-28, (2016).

[26]L. A. Pereira-de-Oliveira, J. P. Castro-Gomes, M. C. S. Nepomuceno, Effect of acrylic fibres geometry on physical, mechanical and durability properties of cement mortars, Construction \& Building Materials Journal, 27(1), pp 189-196, (2012).

[27]C. E. Chalioris, E. F. Sfiri, Shear Performance of Steel Fibrous Concrete Beams, Procedia Engineering, 14, pp 2064-2068, (2011).

[28] M. Hamrat, B. Boulekbache, M. Chemrouk, S. Amziane, Flexural cracking behavior of normal strength, high strength and high strength fiber concrete beams, using Digital Image Correlation technique, Construction \& Building Materials Journal, 106, pp 678-692, (2016).

[29] A. Yazdanbakhsh, S. Altoubat, K. A. Rieder, Analytical study on shear strength of macro synthetic fiber reinforced concrete beams, Engineering Structures Journal, 100, pp 622-632, (2015).

[30]D. R. Sahoo, K. Maran, A. Kumar, Effect of steel and synthetic fibers on shear strength of RC beams without shear stirrups,
Construction \& Building Materials Journal, 83, pp 150-158, (2015).

[31]Jr. S. Furlan, J. B. de Hanai, Shear behaviour of fiber reinforced concrete beams, Cement \& Concrete Composites Journal, 19(4), pp 359-366, (1997). 